\title{
The Academic Division of Breast and Gynaecological Oncology of the Institute for Cancer Research and Treatment (IRCC) of Candiolo, Turin, Italy
}

\author{
Riccardo Ponzone Nicoletta Biglia Riccardo Roagna Luca Sgro Piero Sismondi
}

Academic Division of Breast and Gynaecological Oncology, Institute for Cancer Research and Treatment (IRCC) of Candiolo and AO Ordine Mauriziano, University of Turin, Italy

\section{The Institute for Cancer Research and Treatment of Candiolo}

The mission of the Institute for Cancer Research and Treatment (IRCC, fig. 1) is to make a significant contribution to fight cancer by understanding the basics and by providing optimal diagnostic and therapeutic services. The interaction between experimental and clinical expertise allows constant transfer of innovative biological models into the clinic. Vice versa, non-conventional diagnostic and therapeutic ideas are extended and further developed by basic research. Efforts and resources are concentrated on a number of well-defined pathologies and selected clinical problems. The following units are currently active at the institute: Anaesthesiology and Pain Therapy, Breast and Gynaecological Oncology, Gastroenterology, Laboratory Medicine, Medical Oncology, Pathology, Radiology, Radiation Therapy, Surgical Dermatology and Surgical Oncology.

\section{The Academic Division of Breast and Gynaecological Oncology}

The management of breast cancer cases at our Division is shared with the breast 'Interdisciplinary Group of Cure' (ICG), a structure mainly devoted to the co-ordination of the clinical activities by formal auditing of cases and revision of procedures. The breast IGC has also acquired a role in the integration of the research activities of all the units involved in breast care, such as radiology, pathology, medical oncology and radiotherapy, with an organisation resembling that of the Breast Units operating in the United Kingdom. Further services are available at our joined hospital in the city centre, such as plastic surgery, nuclear medicine and rehabilitation.

\section{KARGER}

Fax +497614520714

Information@Karger.de

www.karger.com (c) 2008 S. Karger GmbH, Freiburg

Accessible online at:

www.karger.com/brc

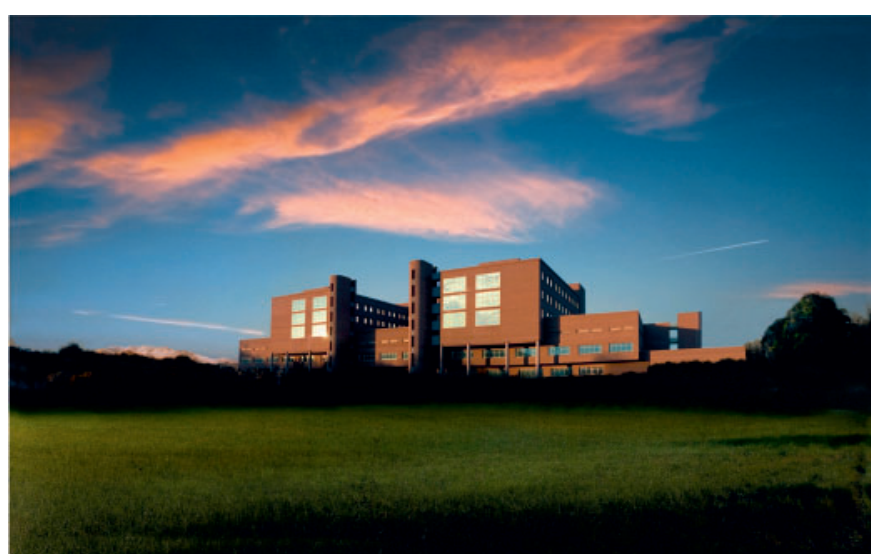

Fig. 1. The Institute for Cancer Research and Treatment (IRCC) of Candiolo, Turin, Italy.

\section{Clinical Activity}

The unit provides outpatient services, such as clinics for symptomatic breast patients (2,500/year), for the follow up of breast cancer patients (3,000/year), for the management of menopausal symptoms in breast cancer survivors (200/year) and for the genetic testing and surveillance of women with suspected/proven hereditary breast cancer (100/year). Weekly interdisciplinary meetings are held to discuss and manage all breast cancer cases. Two separate services of day surgery and regular hospital admittance are provided both at the IRCC, where most of the cases are treated, and at the Mauriziano Hospital if surgery involving plastic reconstruction is required. Overall, approximately 500 new breast cancer patients per year are operated and then provided adjuvant therapies in our centres.

\section{Clinical Research}

Several clinical trials of surgical therapies have been completed over the last years or are currently underway in the fields

Dr. Riccardo Ponzone, MD, PhD

Division of Breast and Gynaecological Oncology

of the Institute for Cancer Research and Treatment (IRCC)

Strada Provinciale 42, Km 3.95, 10060 Candiolo, Turin, Italy

Tel. +39011 9933-444, Fax -447

rponzone@mauriziano.it,www.ircc.it 


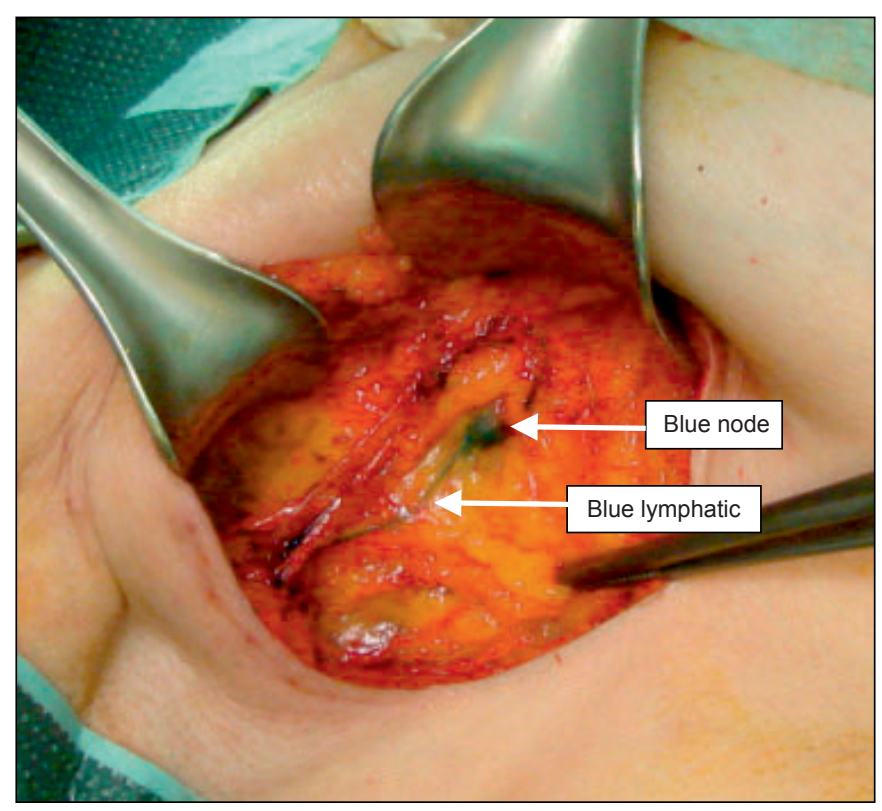

Fig. 2. Axillary Reverse Mapping (ARM): subcutaneous injection of patent blue dye in the inner part of the arm to locate the lymphatic pathway from the arm during axillary dissection.

of conservative oncoplastic breast surgery, sentinel node biopsy and intercostal-brachial nerve preservation during axillary dissection. A new application of the sentinel node technique (axillary reverse mapping) is currently tested to identify and spare the lymphatic pathway of the arm during axillary dissection and sentinel node biopsy (fig. 2). In collaboration with the unit of radiotherapy, we are conducting a trial of accelerated partial breast radiotherapy in conservatively treated breast cancer patients at low risk of local relapse. With the unit of radiology we are assessing the potential of breast MRI (magnetic resonance imaging) for the preoperative evaluation of breast cancer patients of young age and/or dense breasts and for the surveillance of BRCA1/2 mutation carriers. Breast MRI is also tested for the early assessment of tumour response to pre-operative chemotherapy and several trials of hormonal therapies, chemotherapy and targeted biologic therapies are underway in the prevention, adjuvant and metastatic settings in collaboration with the unit of medical oncology. Non-hormonal compounds for menopausal symptoms and vaginal oestrogens for urogenital atrophy, as well as the impact of adjuvant treatments on the cognitive performance and psycho-sexual well-being are tested in breast cancer patients during or after the completion of adjuvant treatments.

\section{Basic Research}

Over the last years the activity of our laboratory has been focused on the genome-wide identification of potential oestrogen-regulated genes in vitro through cDNA microarray analysis, which led to the selection of a group of over 450 genes. A gene expression profile was also identified that associates with the risk of recurrence of primary breast cancer, by microarray analysis and multigene real-time RT-PCR of predefined sets of neoplastic tissues. Among differentially expressed genes, a number of new, previously unidentified genes have been characterised in archival tumour tissues as new prognostic and predictive markers. One of these, ANG-2, was found highly significantly associated to poor clinical outcome, also in lymph nodenegative patients.

\section{Teaching}

The division belongs to the University of Turin Medical School and both medical students and postgraduate fellows are currently working in the clinics, wards and labs.

\section{Breast Interdisciplinary Group of Cure}

Breast Nurses: Annamaria Ballari, Nadia Declame, Annamaria Pellegrino, Teresa Rinarelli, Patrizia Torrisi

Breast and Gynaecological Oncology: Prof. Piero Sismondi, Prof. Nicoletta Biglia, Dr. Riccardo Ponzone, Dr. Riccardo Roagna, Dr. Luca Sgro

Clinical Oncology: Prof. Massimo Aglietta, Dr. Filippo Montemurro, Dr. Giorgio Valabrega, Dr. Stefania Redana

Genetic Counselling: Prof. Barbara Pasini

Pathology: Dr. Mauro Risio, Dr. Giovanni De Rosa, Dr. Ivana Sarotto

Radiology: Dr Daniele Regge, Dr. Lisa Cellini, Dr. Laura Martincich

Radiotherapy: Dr. Pietro Gabriele, Dr. Marco Gatti. 